\title{
Cryptococcus gattii Complex Infections in HIV-Infected Patients, Southeastern United States
}

\author{
Kaylee T. Bruner, ${ }^{1}$ Carlos Franco-Paredes, Andrés F. Henao-Martínez, \\ Gregory M. Steele, Daniel B. Chastain
}

\begin{abstract}
$\underset{\text { MDUCATION }}{\text { Medscape ACTIVITY }}$
In support of improving patient care, this activity has been planned and implemented by Medscape, LLC and Emerging Infectious Diseases. Medscape, LLC is jointly accredited by the Accreditation Council for Continuing Medical Education (ACCME), the Accreditation Council for Pharmacy Education (ACPE), and the American Nurses Credentialing Center (ANCC), to provide continuing education for the healthcare team.

Medscape, LLC designates this Journal-based CME activity for a maximum of 1.00 AMA PRA Category 1 Credit(s) ${ }^{\mathrm{TM}}$. Physicians should claim only the credit commensurate with the extent of their participation in the activity.

All other clinicians completing this activity will be issued a certificate of participation. To participate in this journal CME activity: (1) review the learning objectives and author disclosures; (2) study the education content; (3) take the post-test with a $75 \%$ minimum passing score and complete the evaluation at http://www.medscape.org/journal/eid; and (4) view/print certificate. For CME questions, see page 2140.
\end{abstract}

Release date: October 16, 2018; Expiration date: October 16, 2019

Learning Objectives

Upon completion of this activity, participants will be able to:

- Assess the epidemiology of Cryptococcus gattii complex infections in HIV-infected patients, based on a case series report

- Evaluate the clinical features of C. gattii complex infections in HIV-infected patients, based on a case series report

- Determine the clinical implications of $C$. gattii complex infections in HIV-infected patients, based on a case series report

\section{CME Editor}

Thomas J. Gryczan, MS, Technical Writer/Editor, Emerging Infectious Diseases. Disclosure: Thomas J. Gryczan, MS, has disclosed no relevant financial relationships.

\section{CME Author}

Laurie Barclay, MD, freelance writer and reviewer, Medscape, LLC. Disclosure: Laurie Barclay, MD, has disclosed the following relevant financial relationships: owns stock, stock options, or bonds from Pfizer.

\section{Authors}

Disclosures: Kaylee T. Bruner, PharmD; Carlos Franco-Paredes, MD, MPH; Gregory M. Steele, RN, MSN, FNP-BC; and Daniel B. Chastain, PharmD, have disclosed no relevant financial relationships. Andrés F. Henao-Martínez, MD, has disclosed the following relevant financial relationship: served as an advisor or consultant for Bayer.

Author affiliations: Phoebe Putney Memorial Hospital, Albany, Georgia, USA (K.T. Bruner, G.M. Steele, D.B. Chastain); Hospital Infantil de Mexico, Federico Gomez, Mexico City, Mexico (C. Franco-Paredes); University of Colorado Denver Anschutz Medical Campus, Aurora, Colorado, USA (C. Franco-Paredes, A.F. Henao-Martínez); University of Georgia College of Pharmacy, Albany (D.B. Chastain)

DOI: https://doi.org/10.3201/eid2411.180787
Cryptococcus gattii traditionally infects immunocompetent hosts and causes devastating pulmonary or central nervous system disease. However, this infection rarely occurs in patients infected with HIV. We report 3 cases of HIV-associated C. gattii complex infections in the southeastern United States. Detection of C. gattii in HIV-infected patients in this region warrants increased awareness of this threat to ensure appropriate diagnosis and treatment to optimize patient outcomes.

\footnotetext{
${ }^{1}$ Current affiliation: Archbold Medical Center, Thomasville, Georgia, USA.
} 
C ryptococcus gattii is an encapsulated fungus found primarily in tropical and subtropical regions such as Australia and South America (1). C. gattii was first documented as an emerging pathogen in the United States in 1999 when an outbreak occurred in Oregon and Washington (1). More recently, sporadic cases have been reported throughout the southeastern United States (2-4). Theories for the emergence of $C$. gattii in temperate areas have included climate change, increased travel, and anthropogenic activity; however, the exact mechanism remains unknown $(1,4)$.

$C$. gattii was previously known as variant of $C$. neoformans but was later recognized as an independent species of Cryptococcus (4). Recently, C. gattii was reclassified as a species complex comprised of 4 individual species (5). Similar to $C$. neoformans, $C$. gattii is acquired through inhalation; infection can progress to pneumonia and central nervous system disease by dissemination into the bloodstream. C. gattii has been associated with increased virulence and more severe neurologic manifestations than $C$. neoformans, resulting in major illness and death (3). Although treatment is currently the same as for C. neoformans, it has previously been suggested that more aggressive management of neurologic complications, including decreasing elevated intracranial pressure and possibly early use of dexamethasone, might be warranted for $C$. gattii cases $(2,3)$. In addition, longer duration of induction therapy, averaging approximately 6 weeks, has been required for $C$. gattii infections ( 6 ).

C. gattii infection has traditionally been reported more often in immunocompetent persons, in contrast to C. neoformans, which is more prominent in severely immunocompromised hosts, particularly among those with HIV/ AIDS $(3,7)$. However, more recent evidence has identified some potential risk factors for $C$. gattii meningoencephalitis. These factors include antibodies against granulocytemacrophage colony-stimulating factor, which leads to macrophage dysfunction, and chronic medical conditions, including diabetes mellitus and other illnesses, such as endstage liver or renal disease (8-10).

Cases of C. gattii meningoencephalitis in HIV-infected patients have been reported rarely in areas with high HIV prevalence, such as Botswana and sub-Saharan Africa (11). It appears that the only cases reported of C. gattii in HIV/AIDS patients in the United States have been limited to a small number in southern California (12).

We report 3 cases of $C$. gattii complex meningitis and pneumonitis in HIV-infected patients residing in southwestern Georgia. These cases should alert clinicians for detection of HIV-associated C. gattii complex in the southeastern United States.

\section{Case-Patient 1}

A 34-year-old man with a history of infection with HIV and medication noncompliance was admitted to Phoebe
Putney Memorial Hospital (Albany, GA, USA) because of a 5-week history of nausea, vomiting, and weight loss. He also had headaches, photophobia, and subjective syncope. The patient had a CD4+ T-cell count of 6 cells $/ \mathrm{mm}^{3}$ and an HIV-1 RNA level of 71,265 copies/mL. He reported no recent travel history or exposure to animals. At admission, initial workup included a barium swallow procedure and kidney, ureter, and bladder radiography. These procedures showed no unusual findings.

After we observed an additional syncopal episode, we ordered a test for serum cryptococcal antigen $(\mathrm{CrAg})$ and magnetic resonance imaging (MRI) of the brain because of the HIV status of the patient and concern for an intracranial infectious process. After detection of a serum $\mathrm{CrAg}$ titer $>1: 2,560$, a lumbar puncture (LP) was performed on day 4 of hospitalization. The LP showed an opening pressure of $24 \mathrm{~cm}$ of water, 5 leukocytes $/ \mathrm{mm}^{3}$ ( $6 \%$ polymorphonuclear cells and $94 \%$ mononuclear cells), 0 erythrocytes $/ \mathrm{mm}^{3}$, a protein level of $29 \mathrm{mg} / \mathrm{dL}$, and a glucose level of $49 \mathrm{mg} / \mathrm{dL}$. A positive result (titer $>1: 2,560$ ) was observed for $\mathrm{CrAg}$ in cerebrospinal fluid (CSF).

The patient was given intravenous (IV) amphotericin B lipid complex $(5 \mathrm{mg} / \mathrm{kg} / \mathrm{d})$ and oral flucytosine $(25 \mathrm{mg} /$ $\mathrm{kg} 4 \times / \mathrm{d}$ ). On day 5 , a repeat LP was performed to evaluate intracranial pressure and showed identical opening and closing pressures of $5 \mathrm{~cm}$ of water. After 5 days of treatment with amphotericin B lipid complex and flucytosine, renal dysfunction and thrombocytopenia developed on hospital day 9. The patient was then given oral fluconazole (800 mg 1×/d). Blood and CSF cultures grew Cryptococcus sp., which we further identified as $C$. gattii complex by using matrix-assisted laser desorption/ionization-time of flight mass spectrometry.

MRI of the brain showed enhancement of right frontal lobe adjacent to the lateral ventricle with subtle nodular enhancement within the right caudate head. Nonenhancing T2 and fluid-attenuated inversion recovery MRI showed hyperintensities within bilateral deep nuclei.

After 14 days of antifungal therapy, the patient was deemed stable. He was discharged and received oral fluconazole $(800 \mathrm{mg} 1 \times / \mathrm{d})$. He was scheduled for follow-up in the outpatient clinic 2 weeks later for a repeat LP and initiation of antiretroviral therapy (ART). Unfortunately, the patient did not return for continued care.

\section{Case-Patient 2}

A 47-year-old man with a medical history of hypertension and infection with HIV was admitted to Phoebe Putney Memorial Hospital because of a 2-week history of fever, nausea, headaches, and unsteady gait. Outpatient records showed a CD4+ T-cell count of $<20$ cells $/ \mathrm{mm}^{3}$ and an HIV1 RNA level of 1,653 copies $/ \mathrm{mL}$, for which he was recently given ART. This therapy consisted of emtricitabine (200 
$\mathrm{mg} 1 \times / \mathrm{d}$ ), tenofovir disoproxil fumarate $(300 \mathrm{mg} 1 \times / \mathrm{d}$ ), raltegravir (400 mg $2 \times / \mathrm{d}$ ), and etravirine (200 mg $2 \times / \mathrm{d})$.

MRI of the brain performed at admission was unremarkable, with no definitive evidence of acute ischemic, intracranial hematoma, or enhancing intracranial lesion. Initially, the patient was given levofloxacin for treatment of possible sinusitis, but he continued to experience intermittent episodes of fever and persistent headaches. On day 2 after admission, an LP was performed and showed increased opening pressure, 85 leukocytes $/ \mathrm{mm}^{3}$ (1\% polymorphonuclear cells and 99\% mononuclear cells), 11 erythrocytes $/ \mathrm{mm}^{3}$, a protein level of 96 $\mathrm{mg} / \mathrm{dL}$, and glucose level of $42 \mathrm{mg} / \mathrm{dL}$. A positive result (titer $>1: 256$ ) was observed for CrAg in CSF.

The positive finding for $\mathrm{CrAg}$ prompted initiation of induction therapy for cryptococcal meningitis, which consisted of IV liposomal amphotericin B (5 mg/ $/ \mathrm{kg} / \mathrm{d})$ and oral flucytosine $(25 \mathrm{mg} / \mathrm{kg} 4 \times / \mathrm{d})$. CSF cultures grew yeast, which we eventually identified as $C$. gattii complex by using 1-canavanine, glycine, bromothymol blue (CGB) agar. Blood cultures remained sterile throughout hospitalization. The patient was given IV dexamethasone (4 mg every 6 h) because of recurrent headaches. Before completion of 14 days of induction therapy, a repeat LP was performed and showed an opening pressure of $43 \mathrm{~cm}$ of water, a closing pressure of $30 \mathrm{~cm}$ of water, 97 leukocytes $/ \mathrm{mm}^{3}(100 \%$ mononuclear cells), 4 erythrocytes $/ \mathrm{mm}^{3}$, a protein level of $89 \mathrm{mg} / \mathrm{dL}$, and a glucose level of $58 \mathrm{mg} / \mathrm{dL}$. CSF remained positive for $\mathrm{CrAg}$, but the titer decreased to $1: 16$, and the CSF culture remained sterile.

The patient was discharged and received voriconazole and a dexamethasone taper over a 6-week period. Voriconazole was continued for 6 months and was chosen for the consolidation phase of treatment because there is some evidence that the MIC of voriconazole is lower than the MIC of fluconazole for most C. gattii complex isolates $(13,14)$. During the subsequent 5 years of follow-up, his ART was changed to abacavir (300 mg $2 \times / \mathrm{d})$, lamivudine $(150 \mathrm{mg}$ $2 \times / \mathrm{d}$ ), and raltegravir (400 $\mathrm{mg} 2 \times / \mathrm{d}$ ). The patient continued secondary prophylaxis with oral voriconazole until CD4+
T-cell counts were $\geq 100$ cells $/ \mathrm{mm}^{3}$ for at least 3 months. Recent laboratory results showed undetectable HIV-1 RNA and a CD4+ T-cell count of 256 cells $/ \mathrm{mm}^{3}$.

\section{Case-Patient 3}

A 47-year-old man with a history of depression and infection with HIV for $\approx 20$ years was admitted to Phoebe Putney Memorial Hospital because of suicidal ideation caused by persistent pain. He also reported body aches, fatigue, and weakness for a 1-month duration, but denied pulmonary symptoms or fevers. He was previously receiving ART, but had been noncompliant for the previous 2 years because of homelessness.

At admission, initial examination showed a CD4+ Tcell count of 26 cells $/ \mathrm{mm}^{3}$, an HIV-1 RNA level of 272,152 copies/mL, positive result for serum CrAg (titer 1:160), and a right hilar mass by chest radiograph. The patient was given IV amphotericin B lipid complex $(5 \mathrm{mg} / \mathrm{kg} / \mathrm{d})$ and oral flucytosine $(25 \mathrm{mg} / \mathrm{kg} 4 \times / \mathrm{d})$. An LP showed 3 leukocytes $/ \mathrm{mm}^{3}$ (100\% mononuclear cells), 4 erythrocytes $/ \mathrm{mm}^{3}$, a protein level of $42 \mathrm{mg} / \mathrm{dL}$, and a glucose level of $62 \mathrm{mg} /$ $\mathrm{dL}$. The patient had a negative result for $\mathrm{CrAg}$ in CSF.

Because of these findings, we performed computed tomography (CT) of the chest and identified a mass $(4.0 \mathrm{~cm}$ $\times 2.5 \mathrm{~cm})$ with central cavitation in the right lower lobe abutting the pleural surface (Figure). The patient underwent a CT-guided lung biopsy to evaluate the right lower lobe mass. Pathologic examination demonstrated fibrous tissue and numerous cystic spaces containing macrophages and variably sized organisms with a thick capsule that were strongly positive when stained with Grocott's methanamine silver special stain. The morphologic features were consistent with those of a Cryptococcus sp. We observed many budding yeasts by using Gram stain.

The patient continued to receive oral flucytosine (25 $\mathrm{mg} / \mathrm{kg} 4 \times / \mathrm{d}$ ), but because of an acute kidney injury after 3 days, amphotericin B lipid complex was replaced with IV fluconazole ( $800 \mathrm{mg}$ in a single dose), followed by oral fluconazole $(400 \mathrm{mg} 1 \times / \mathrm{d})$. He remained hospitalized for an

Figure. Computed tomography images of the chest of an HIV-infected 47-year-old man (case-patient 3) with Cryptococcus gattii complex infection, southeastern United States. Transverse (A) and frontal (B) views without intravenous contrast showed a mass (arrows) $(4.0 \mathrm{~cm} \times 2.5$ $\mathrm{cm}$ ) that had central cavitation posteriorly in the right lower lobe abutting the pleural surface. The central cavitary portion of this lesion had a
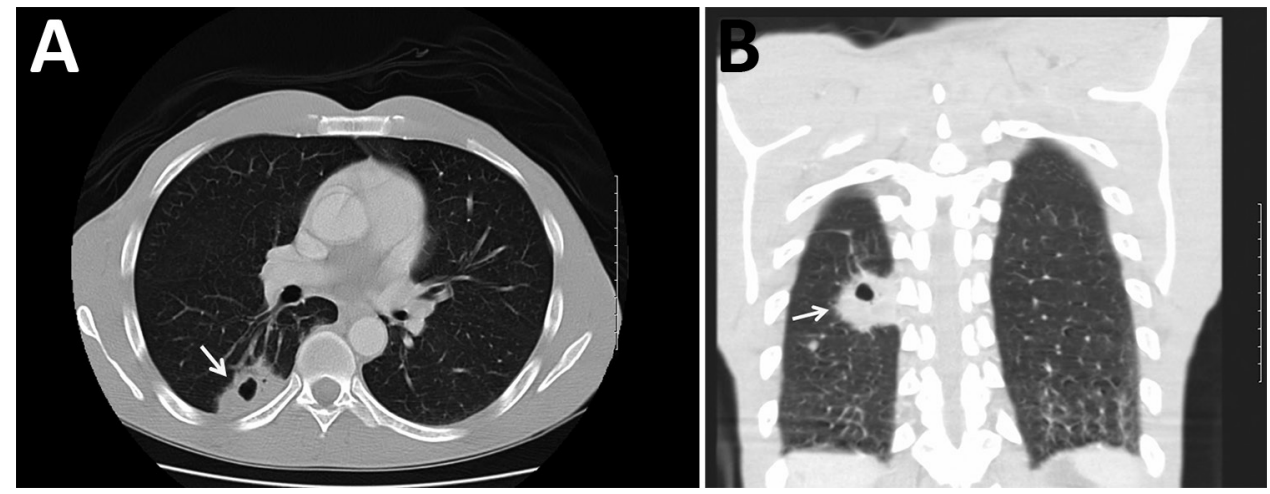
maximum length of $\approx 1.3 \mathrm{~cm}$ and no evidence of fluid level or internal soft tissues. 
additional 3 days until growth on CGB agar was identified as $C$. gattii complex. At that time, the patient had improved considerably and was discharged.

During outpatient treatment, the patient received emtricitabine $(200 \mathrm{mg} / \mathrm{d})$, dolutegravir $(50 \mathrm{mg} / \mathrm{d})$, darunavir $(800 \mathrm{mg} / \mathrm{d})$, and cobicistat $(150 \mathrm{mg} / \mathrm{d})$. A CT of his abdomen and pelvis to evaluate lower abdominal pain was performed $\approx 6$ months later and showed resolution of the initial mass. We detected a new 20-mm lesion within the right lower lobe, which was not observed in previous studies. The patient continued to receive fluconazole (400 mg $1 \times /$ d) for an additional 12 months without subsequent radiographic monitoring. His most recent laboratory results showed a CD4+ T-cell count of 250 cells $/ \mathrm{mm}^{3}$ and an HIV-1 RNA level $<40$ copies $/ \mathrm{mL}$ while receiving the aforementioned ART.

\section{Discussion}

We report 3 cases of C. gattii complex infections in HIVinfected patients in the southeastern United States (southwestern Georgia). Given the increasing recognition of $C$. gattii complex in southwestern Georgia, it is routine practice at our institution to plate cryptococcal isolates on CGB agar to distinguish between $C$. neoformans and $C$. gattii complex $(4,15)$. Since implementation of this practice in 2012, of 30 clinical isolates of Cryptococcus sp., 3 (10\%) have been identified as the $C$. gattii complex. Although the environmental source of these infections in southwestern Georgia remains unknown because the 3 patients in this case series had no previous travel, we believe that their immunocompromised state characterized by low CD4+ T-cell counts is a contributing factor for acquiring infection with C. gattii complex.

Historically, most cases of infection with C. gattii were identified in patients who were immunocompetent or otherwise healthy and lived in the Pacific Northwest region of the United States because of endemicity of this fungi to this region (3). However, recent data suggest a longer duration of endemicity in the southeastern United States than in the Pacific Northwest, specifically because of the $C$. gattii VGI-SE clade $(16,17)$.

Data from the 1960s and 1980s indicated detection of C. neoformans serotypes B and $\mathrm{C}$, now recognized as $C$. gattii, from clinical and environmental isolates. However, the first cases of $C$. gattii infections from regions outside of the Pacific Northwest were reported in 2009. In a retrospective database review by the Centers for Disease Control and Prevention for 2004-2011, a total of 96 case-patients infected with $C$. gattii were identified; 81 resided in the Pacific Northwest (Washington and Oregon) and most were infected with the VGII outbreak strain, which was acquired from the outbreak on Vancouver Island in Canada (10). Nonoutbreak strains VGI and VGIII were confined to HIV-negative persons residing in states outside the Pacific Northwest in comparison with outbreak case-patients, of whom 3 were infected with HIV. Similar to the findings for case-patient 3 in our case report, 59\% of patients in the Pacific Northwest outbreak had C. gattii pneumonia; however, $75 \%$ had respiratory symptoms, but case-patient 3 had no respiratory symptoms (10).

In 2010, 276 isolates of Cryptococcus sp. in HIVinfected patients were reported in southern California, of which 34 isolates were identified as C. gattii, demonstrating that $C$. gattii had been present previously in immunocompromised patients (12). More recently, 25 case-patients outside the Pacific Northwest have been reported; 5 had C. gattii pulmonary disease, 12 had central nervous system disease, and 8 had a combination of the 2 C. gattii diseases (2). Of the 25 case-patients reported, 7 resided in the southeastern states: 1 in Florida, 5 in Georgia, and 1 in Alabama. The HIV status of these case-patients was not reported; however, the case-patient in Florida was an otherwise healthy native of Florida who had no known travel to areas to which $C$. gattii is endemic (18). Similar to casepatients 1 and 2, two previous case-patients with $C$. gattii meningitis were reported in southwestern Georgia $(3,15)$. The surprising difference was that these patients were HIV negative and otherwise healthy.

Speciation of cryptococcal isolates is essential because C. gattii complex meningoencephalitis might have more severe clinical manifestations requiring aggressive management of intracranial hypertension with early evacuation of CSF and treatment with steroids (3). Use of dexamethasone for treatment of $C$. gattii complex meningitis has been associated with positive outcomes by potentially decreasing cerebral edema and tissue damage $(19,20)$. In a small retrospective study of 16 patients in Papua New Guinea infected with $C$. gattii, most patients who received corticosteroids had reduced rates of vision loss and blindness (21). In addition, case-patients infected with the $C$. gattii complex might require prolonged courses of amphotericin B-based therapy compared with case-patients infected with $C$. neoformans (6). Finally, there appears to be different antifungal susceptibility to azoles that might suggest the use of voriconazole instead of fluconazole for the consolidation phase of antifungal therapy $(13,14)$.

Early identification of the $C$. gattii complex can be challenging because of limited accessibility of tests that differentiate Cryptococcus spp., including use of CGB agar (22). Because of lack of availability of testing in local microbiology laboratories, it is likely that infection with $C$. gattii complex is underreported.

On the basis of the cases presented in this report and continued detection of $C$. gattii in the southeastern United States, we believe routine testing to differentiate $C$. neoformans from $C$. gattii is warranted and further investigation is 
required to determine appropriate treatment in comparison with treatment for $C$. neoformans. In addition, healthcare providers should be aware of this pathogen and its potential to cause devastating disease in immunocompromised and immunocompetent patients.

\section{About the Author}

Dr. Bruner is a clinical pharmacist in the Emergency Department, Archbold Medical Center, Thomasville, GA. Her research interests are targeted temperature management, infectious diseases, and emergency medicine.

\section{References}

1. Datta K, Bartlett KH, Baer R, Byrnes E, Galanis E, Heitman J, et al.; Cryptococcus gattii Working Group of the Pacific Northwest. Spread of Cryptococcus gattii into Pacific Northwest region of the United States. Emerg Infect Dis. 2009;15:1185-91. http://dx.doi.org/10.3201/eid1508.081384

2. Harris JR, Lockhart SR, Sondermeyer G, Vugia DJ, Crist MB, D'Angelo MT, et al. Cryptococcus gattii infections in multiple states outside the US Pacific Northwest. Emerg Infect Dis. 2013;19:1620-6. http://dx.doi.org/10.3201/eid1910.130441

3. Franco-Paredes C, Womack T, Bohlmeyer T, Sellers B, Hays A, Patel K, et al. Management of Cryptococcus gattii meningoencephalitis. Lancet Infect Dis. 2015;15:348-55. http://dx.doi.org/ 10.1016/S1473-3099(14)70945-4

4. Harris J, Lockhart S, Chiller T. Cryptococcus gattii: where do we go from here? Med Mycol. 2012;50:113-29. http://dx.doi.org/10.31 09/13693786.2011.607854

5. Hagen F, Khayhan K, Theelen B, Kolecka A, Polacheck I, Sionov E, et al. Recognition of seven species in the Cryptococcus gattii/Cryptococcus neoformans species complex. Fungal Genet Biol. 2015;78:16-48. http://dx.doi.org/10.1016/j.fgb.2015.02.009

6. Chen SC, Korman TM, Slavin MA, Marriott D, Byth K, Bak N, et al.; Australia and New Zealand Mycoses Interest Group (ANZMIG) Cryptococcus Study. Antifungal therapy and management of complications of cryptococcosis due to Cryptococcus gattii. Clin Infect Dis. 2013;57:543-51. http://dx.doi.org/10.1093/cid/cit341

7. Mitchell DH, Sorrell TC, Allworth AM, Heath CH, McGregor AR, Papanaoum K, et al. Cryptococcal disease of the CNS in immunocompetent hosts: influence of cryptococcal variety on clinical manifestations and outcome. Clin Infect Dis. 1995;20: 611-6. http://dx.doi.org/10.1093/clinids/20.3.611

8. Brouwer AE, Siddiqui AA, Kester MI, Sigaloff KC, Rajanuwong A, Wannapasni S, et al. Immune dysfunction in HIV-seronegative, Cryptococcus gattii meningitis. J Infect. 2007;54:e165-8. http://dx.doi.org/10.1016/j.jinf.2006.10.002

9. Cheng PY, Sham A, Kronstad JW. Cryptococcus gattii isolates from the British Columbia cryptococcosis outbreak induce less protective inflammation in a murine model of infection than Cryptococcus neoformans. Infect Immun. 2009;77:4284-94. http://dx.doi.org/10.1128/IAI.00628-09

10. Harris JR, Lockhart SR, Debess E, Marsden-Haug N, Goldoft M, Wohrle R, et al. Cryptococcus gattii in the United States: clinical aspects of infection with an emerging pathogen. Clin Infect Dis. 2011;53:1188-95. http://dx.doi.org/10.1093/cid/cir723

11. Steele KT, Thakur R, Nthobatsang R, Steenhoff AP, Bisson GP. In-hospital mortality of HIV-infected cryptococcal meningitis patients with C. gattii and C. neoformans infection in Gaborone, Botswana. Med Mycol. 2010;48:1112-5. http://dx.doi.org/10.3109/13693781003774689

12. Chaturvedi S, Dyavaiah M, Larsen RA, Chaturvedi V. Cryptococcus gattii in AIDS patients, southern California. Emerg Infect Dis. 2005;11:1686-92. http://dx.doi.org/10.3201/eid1111.040875

13. Lockhart SR, Iqbal N, Bolden CB, DeBess EE, Marsden-Haug N, Worhle R, et al.; Cryptococcus gattii PNW Public Health Working Group. Epidemiologic cutoff values for triazole drugs in Cryptococcus gattii: correlation of molecular type and in vitro susceptibility. Diagn Microbiol Infect Dis. 2012;73:144-8. http://dx.doi.org/10.1016/j.diagmicrobio.2012.02.018

14. Espinel-Ingroff A, Aller AI, Canton E, Castañón-Olivares LR, Chowdhary A, Cordoba S, et al. Cryptococcus neoformansCryptococcus gattii species complex: an international study of wild-type susceptibility endpoint distributions and epidemiological cutoff values for fluconazole, itraconazole, posaconazole, and voriconazole. Antimicrob Agents Chemother. 2012;56:5898-906. http://dx.doi.org/10.1128/AAC.01115-12

15. Sellers B, Hall P, Cine-Gowdie S, Hays AL, Patel K, Lockhart SR, et al. Cryptococcus gattii: an emerging fungal pathogen in the Southeastern United States. Am J Med Sci. 2012;343:510-1. http://dx.doi.org/10.1097/MAJ.0b013e3182464bc7

16. Lockhart SR, Iqbal N, Harris JR, Grossman NT, DeBess E, Wohrle R, et al. Cryptococcus gattii in the United States: genotypic diversity of human and veterinary isolates. PLoS One. 2013;8:e74737. http://dx.doi.org/10.1371/journal.pone.0074737

17. Lockhart SR, Roe CC, Engelthaler DM. Whole-genome analysis of Cryptococcus gattii, southeastern United States. Emerg Infect Dis. 2016;22:1098-101. http://dx.doi.org/10.3201/eid2206.151455

18. Kunadharaju R, Choe U, Harris JR, Lockhart SR, Greene JN. Cryptococcus gattii, Florida, USA, 2011. Emerg Infect Dis. 2013;19:519-21. http://dx.doi.org/10.3201/eid1903.121399

19. Lane M, McBride J, Archer J. Steroid responsive late deterioration in Cryptococcus neoformans variety gattii meningitis. Neurology. 2004;63:713-4. http://dx.doi.org/10.1212/01.WNL.0000134677. 29120.62

20. Phillips P, Chapman K, Sharp M, Harrison P, Vortel J, Steiner T, et al. Dexamethasone in Cryptococcus gattii central nervous system infection. Clin Infect Dis. 2009;49:591-5. http://dx.doi.org/ 10.1086/603554

21. Seaton RA, Verma N, Naraqi S, Wembri JP, Warrell DA. The effect of corticosteroids on visual loss in Cryptococcus neoformans var. gattii meningitis. Trans R Soc Trop Med Hyg. 1997;91:50-2. http://dx.doi.org/10.1016/S0035-9203(97)90393-X

22. Klein KR, Hall L, Deml SM, Rysavy JM, Wohlfiel SL, Wengenack NL. Identification of Cryptococcus gattii by use of L-canavanine glycine bromothymol blue medium and DNA sequencing. J Clin Microbiol. 2009;47:3669-72. http://dx.doi.org/ 10.1128/JCM.01072-09

Address for correspondence: Daniel B. Chastain, University of Georgia College of Pharmacy, 1000 Jefferson St, Albany, GA 31701, USA; email: daniel.chastain@uga.edu 